\title{
Effect of false lumen partial thrombosis on repaired acute type A aortic dissection
}

\author{
Meng-Ta Tsai, MD, ${ }^{\mathrm{a}}$ Hsuan-Yin Wu, MD, ${ }^{\mathrm{a}, \mathrm{b}}$ Jun-Neng Roan, MD, ${ }^{\mathrm{a}, \mathrm{c}}$ Yi-Shan Tsai, MD, ${ }^{\mathrm{d}}$ \\ Patrick C. H. Hsieh, MD, PhD, ${ }^{\mathrm{a}, \mathrm{c}, \mathrm{e}}$ Yu-Jen Yang, MD, and Chwan-Yau Luo, MD, MSc ${ }^{\mathrm{a}, \mathrm{e}}$
}

Objective: Studies on the partial thrombosis of a false lumen after repairing a type A acute aortic dissection (TAAAD) have reported conflicting results. We investigated the effects of a partially thrombosed false lumen on the segmental growth rates, distal aortic reoperations, and long-term survival.

\begin{abstract}
Methods: The postoperative computed tomography scans of 67 patients were retrospectively reviewed. A false lumen was independently defined at 3 segments of the descending thoracic aorta (DTA) on the last follow-up computed tomography scan: the proximal segment near the aortic arch, the distal segment near the diaphragm, and the middle segment.
\end{abstract}

Results: The segmental aortic growth rate of completely thrombosed, completely patent, and partially thrombosed false lumens was $-0.10 \pm 0.31,0.09 \pm 0.22$, and $0.35 \pm 0.60 \mathrm{~mm} / \mathrm{mo}$ at the proximal DTA $(P=.001),-0.04 \pm 0.18,0.12 \pm 0.19$, and $0.28 \pm 0.28 \mathrm{~mm} / \mathrm{mo}$ at the middle DTA $(P<.001)$, and -0.02 $\pm 0.13,0.07 \pm 0.07$, and $0.16 \pm 0.14 \mathrm{~mm} / \mathrm{mo}$ at the distal DTA $(P<.001)$, respectively. The corresponding freedom from reoperation rates for the proximal DTA at 10 years were $100 \%, 88 \%$, and $62 \%(P=.013)$. The overall 10-year survival rate was $89 \%$ and was not significantly different among the study groups.

Conclusions: Partial thrombosis at each segment of a residual false lumen after TAAAD repair correlated with a faster regional aortic growth rate and predicted a greater reoperation rate but did not affect long-term overall survival. (J Thorac Cardiovasc Surg 2014;148:2140-6)

Supplemental material is available online.

Type A acute aortic dissection (TAAAD) remains a challenge to cardiovascular surgeons. Patients who survive the initial operation still face the risks of a residual dissected aorta, aneurysmal dilatation, and rupture. The adverse effects of a patent false lumen and the protective role of a thrombosed false lumen on aortic growth, reoperation, and late mortality seemed to have been generally agreed on. ${ }^{1-9}$ The predisposing effect of partial thrombosis on aortic growth and adverse late outcomes after type B acute aortic dissection (TBAAD) $)^{10,11}$ or surgically

From the Division of Cardiovascular Surgery, ${ }^{a}$ Department of Surgery, National Cheng Kung University Hospital, Tainan, Taiwan; Division of Cardiovascular

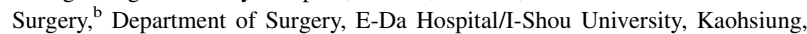
Taiwan; Institute of Clinical Medicine, ${ }^{\mathrm{c}}$ National Cheng Kung University Hospital and College of Medicine, Tainan, Taiwan; Department of Radiology, ${ }^{\mathrm{d}}$ National Cheng Kung University Hospital, Tainan, Taiwan; and Cardiovascular Research Center, ${ }^{e}$ National Cheng Kung University, National Cheng Kung University Hospital, Tainan, Taiwan.

Disclosures: Authors have nothing to disclose with regard to commercial support. Received for publication Oct 30, 2013; revisions received Jan 1, 2014; accepted for publication Feb 4, 2014; available ahead of print March 20, 2014

Address for reprints: Chwan-Yau Luo, MD, MSc, Department of Surgery, National

Cheng Kung University College of Medicine, 138 Sheng-Li Rd, Tainan 704,

Taiwan (E-mail: luochwya@mail.ncku.edu.tw).

$0022-5223 / \$ 36.00$

Copyright $($ C 2014 by The American Association for Thoracic Surgery

http://dx.doi.org/10.1016/j.jtcvs.2014.02.003 repaired TAAAD ${ }^{12}$ has been suggested but has been inconsistent in other reports. ${ }^{9,13}$

The inconsistent results of these studies could be attributable to the diverse presentations and different definitions of partial thrombosis. However, we hypothesized that a residual partially thrombosed false lumen of the descending thoracic aorta (DTA) after surgically repaired TAAAD would be a predisposing factor of the aortic growth rate and adverse late outcomes. We analyzed postoperative computed tomography (CT) images of our patients to determine whether their segmental false lumen status would correlate with the aortic growth rate, reoperation, and overall survival.

\section{METHODS}

\section{Patients and Follow-up}

The ethics committee of our hospital approved our retrospective study. A total of 215 consecutive patients underwent surgery for TAAAD from October 1989 through December 2012. The overall 30-day mortality rate was $24.7 \%$ (53 patients) and had decreased to $12.9 \%$ between 2003 and 2012. The patients were routinely given postoperative $\beta$-blockers and calcium channel blockers, unless these were contraindicated. A CT scan for each patient was planned for 3 to 6 months postoperatively and annually thereafter. If the aortic size was stationary after 3 consecutive annual follow-up examinations, the follow-up interval for CT was changed to every 2 years. Some patients who had undergone surgery before 2000 underwent their first postoperative CT scan after the first postoperative year, but all had adhered to the same CT protocol subsequently. We included 67 of the 155 survival-to-discharge patients who had undergone $\geq 2$ postoperative CT scans $>6$ months apart before reoperation or death. 


\section{Abbreviations and Acronyms \\ CT = computed tomography \\ DTA $=$ descending thoracic aorta \\ TAAAD $=$ type $\mathrm{A}$ acute aortic dissection \\ $\mathrm{TBAAD}=$ type $\mathrm{B}$ acute aortic dissection}

We excluded those patients who had undergone magnetic resonance imaging or noncontrast CT because of renal insufficiency, those with incomplete tests, and those with DeBakey type II TAAAD.

\section{Operative Procedure}

We used femoral artery cannulation from 1989 to 2006, with deep hypothermia circulatory arrest and retrograde cerebral perfusion for brain protection. From late 2006 onward, right axillary artery cannulation using an 8-mm side-arm graft was routinely used for cardiopulmonary bypass and selective antegrade cerebral perfusion. After the patient's body had been cooled to $20^{\circ} \mathrm{C}$, deep hypothermia circulatory arrest was begun. The brain was protected using retrograde cerebral perfusion to maintain a pressure not exceeding 25 to $30 \mathrm{~mm} \mathrm{Hg}$ or selective antegrade cerebral perfusion with a flow of $10 \mathrm{~mL} / \mathrm{kg} / \mathrm{min}$ to maintain a right radial arterial pressure of 40 to $70 \mathrm{~mm} \mathrm{Hg}$. The ascending aorta and aortic arch were examined and trimmed until the entry site was excluded, if possible, and it was reinforced with Teflon felt. Open distal aortic anastomosis was used. If the entry site was located in the ascending aorta only, an ascending aorta or hemiarch replacement was done. If the entry site was located in the aortic arch or the arch vessels were compromised, subtotal or total arch replacement was done. The proximal reconstruction was performed last when whole body perfusion had resumed and systemic rewarming had begun.

\section{CT Protocol}

All patients were followed up within our institute. Except for the early postoperative CT images of those patients included in earlier period, the protocol for all CT scans protocol was uniform, especially for the last follow-up CT scan that we used to categorize the false lumen status. The CT images were acquired using a 16-slice multidetector CT (Somatom Emotion, Siemens, Munich, Germany) until May 2010 and then using a second-generation dual-source CT (Definition Flash, Siemens). We used $80 \mathrm{~mL}$ of contrast medium after a 30-mL normal saline flash with a power injection at a high flow rate $(3-3.5 \mathrm{~mL} / \mathrm{s})$. The arterial phase was scanned using a bolus-tracking technique with the region-of-interest placed on the aortic root. When the CT density in the aortic root reached $>100 \mathrm{HU}$, the scan was started with a 5-second delay on the multidetector CT scanner (scan pitch, 1.5) and a 10-second delay on the dual-source CT (scan pitch, 2.0). The collimator was $1.5 \mathrm{~mm}$ on the multidetector $\mathrm{CT}$ and $0.6 \mathrm{~mm}$ on the dual-source CT scanners. The delayed phase was acquired within 60 to 70 seconds after the contrast medium had begun. Axial and oblique sagittal images that paralleled the aortic arch were reformatted to a 5-mm slice thickness. We did not arrange contrast CT for patients with a creatinine level of $>2 \mathrm{mg} / \mathrm{dL}$. If the creatinine level was 1.5 to $2 \mathrm{mg} / \mathrm{dL}$, we did not adjust our contrast protocol.

\section{Segmental Analysis of Postoperative Growth Rate and False Lumen Status}

Previous studies ${ }^{9-11,13,14}$ have generally categorized the residual false lumen according to the entire false lumen status. However, it is likely that a partial thrombosis of the residual dissected descending aorta will present differently, with different thrombi lengths, distribution, and associated inlets and outlets, and could result in a different fate for each corresponding segment. Therefore, we hypothesized that the significance of a partially thrombosed false lumen on the postoperative growth rate and late outcome would be better clarified using segmental analysis (Figure E1).

Because dilatation of the distal aorta after repaired TAAAD has been reported to be at the DTA, ,2,6,13,14 we selected 3 levels for a segmental analysis of the growth rate and false lumen status: the proximal DTA distal to the left subclavian artery and aortic arch (Figure E1, $A$ ), the distal DTA at the level of the diaphragm crura (Figure E1, $C$ ), and the middle DTA (Figure 1, $B$ ). By defining the axial CT image where the left subclavian artery immediately disappeared from the arch as the first slice and the axial CT image where the diaphragm crura appeared as the last, the $3 \mathrm{seg}$ ments were divided into 3 equal sizes according to the number of slices. The maximal aortic diameter perpendicular to the aortic axis was measured in the axial view of each segment. The aortic growth rate in each segment was calculated by dividing the size difference between the first postoperative image and the last follow-up image by the interval between them $(\mathrm{mm} /$ mo). The image before reoperation or death was used as the last follow-up image for the patients who had undergone reoperation or who had died.

The false lumen status was categorized and defined independently in each segment using the delayed-phase image. We used the last follow-up CT scan to evaluate the false lumen status, instead of the first postoperative CT scan. This strategy ${ }^{13}$ was chosen because the false lumen status dynamically changes during follow-up. ${ }^{2}$ The false lumen status determined using the last CT scan will reflect, not only the perioperative effects, but also the postoperative long-term medical management. A false lumen homogeneously contrast enhanced was recognized as "completely patent." A false lumen with a thrombus in any part of it with limited flow was considered "partially thrombosed." A false lumen completely lacking blood flow was defined as "completely thrombosed." The false lumen status was judged independently in all 3 DTA segments.

\section{Relationship Between False Lumen Status and Late Outcomes}

Patients who underwent reoperation on for aneurysms $>6 \mathrm{~cm}$ or for rupture in the distal aorta were included. Reoperations for complications of the proximal aorta ( 2 of $67,3.0 \%$; eg, graft infection, aortic regurgitation, and root dilatation) were excluded. The reoperations were assigned to each aortic segment group according to the segment requiring reoperation for an analysis of its relationship with the false lumen status at the last follow-up CT scan. The overall survival rate was compared between the false lumen groups and the aortic segments.

\section{Dynamic Change of False Lumen Status}

The false lumen status at the first postoperative and serial follow-up CT scans was compared. Excluding the 13 patients (19.4\%) from the earlier period, whose first postoperative CT scan had occurred after postoperative year 1, the incidence and timing of the change in the false lumen status in the other 54 patients $(80.6 \%)$ were investigated.

\section{Statistical Analysis}

All data are presented as the mean \pm standard deviation. Multigroup comparisons used analysis of variance. Freedom from late reoperation or death was estimated using the nonparametric actuarial Kaplan-Meier curve, which was stratified according to the false lumen status at each level. The Statistical Package for Social Sciences, version 17.0.0, for Windows (SPSS, Chicago, Ill), was used for all statistical analyses.

\section{RESULTS}

Patient Demographics, Procedures, and Intraoperative Findings

A total of 67 patients were included in the present study. Their mean age was $52.96 \pm 12.08$ years, and 50 were men 


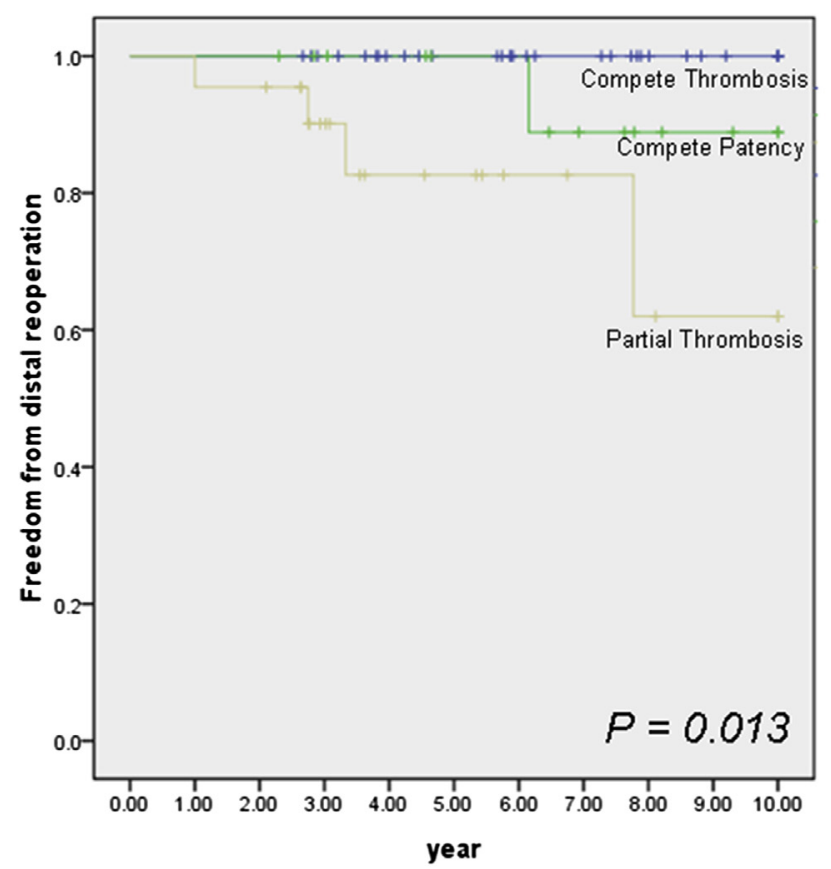

Patients at risk (freedom rate\%)

\begin{tabular}{ccccc} 
& year & 1 & 5 & 10 \\
\cline { 3 - 5 } & Compete Thrombosis & $31(100)$ & $20(100)$ & $4(100)$ \\
& Compete Patency & $14(100)$ & $9(100)$ & $2(88)$ \\
A & Partial Thrombosis & $22(95)$ & $8(83)$ & $2(62)$ \\
\cline { 2 - 5 }
\end{tabular}

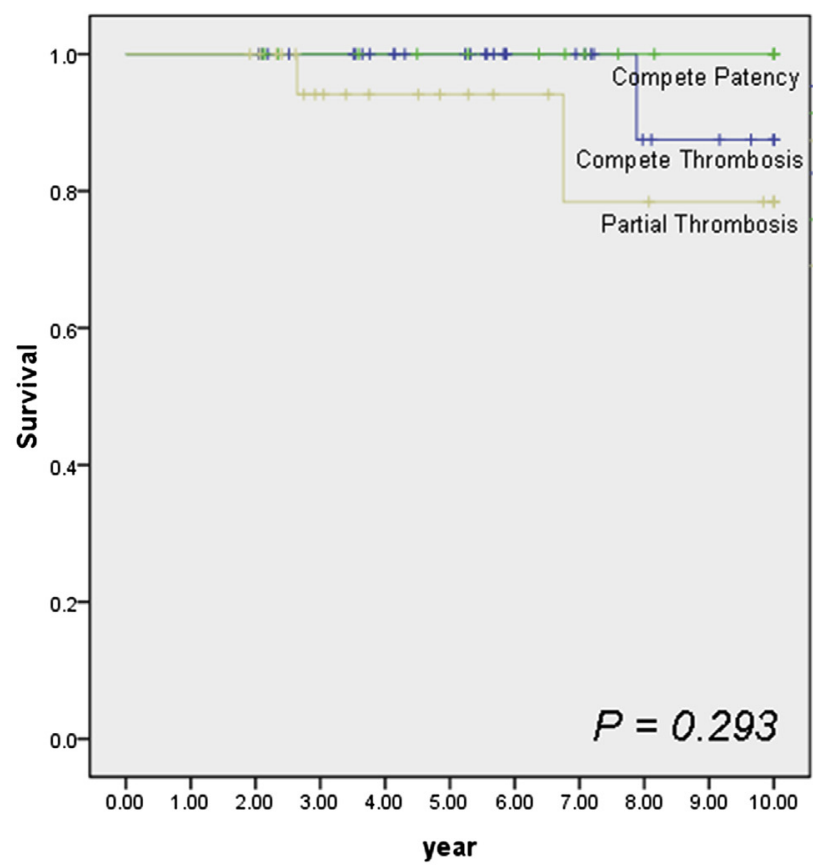

Patients at risk (survival rate\%)

\begin{tabular}{ccccc} 
& year & 1 & 5 & 10 \\
\hline Compete Thrombosis & $31(100)$ & $20(100)$ & $3(89)$ \\
& Compete Patency & $14(100)$ & $9(100)$ & $3(100)$ \\
B & Partial Thrombosis & $22(100)$ & $9(94)$ & $3(80)$ \\
\hline
\end{tabular}

FIGURE 1. Kaplan-Meier curves for (A) freedom from distal aortic reoperation and (B) overall survival stratified by false lumen status according to the proximal descending thoracic aorta segment.

(74.6\%). Of the 67 patients, $49(73.1 \%)$ had a history of hypertension, and 26 (38.8) had preoperative malperfusion (Table E1). Of the 67 patients, $42(62.7 \%)$ underwent ascending aorta replacement with or without hemiarch replacement and $25(37.3 \%)$ ascending aorta replacement and subtotal or total arch replacement, including $4(6.0 \%)$ elephant trunk reconstructions. Three patients $(4.5 \%)$ required coronary artery bypass and $4(6.0 \%)$ femoralfemoral bypass during the initial operation. One patient $(1.5 \%)$ was given a superior mesenteric artery stent on postoperative day 4 because of persistent bowel malperfusion.

\section{Segmental Aortic Growth Rate According to Segmental Analysis of False Lumen Status}

The mean follow-up time was $6.28 \pm 4.01$ years (range, 1.91-18.77). The average interval between the 2 CT scans was $40.18 \pm 23.99$ months. The distribution of the cases among the 3 false lumen subgroups at each aortic segment was different, because the false lumen status was defined independently for all 3 DTA segments. The distribution of the complete thrombosis, completely patent, and partial thrombosis subgroups in the proximal segment of the
DTA was $46.3 \%, 20.9 \%$, and $32.8 \%$, respectively (Table 1).

The segmental aortic growth rate of the completely thrombosed, completely patent, and partially thrombosed false lumens was $-0.10 \pm 0.31,0.09 \pm 0.22$, and $0.35 \pm$ $0.60 \mathrm{~mm} / \mathrm{mo}$ at the proximal DTA $(P=.001),-0.04 \pm$ $0.18,0.12 \pm 0.19$, and $0.28 \pm 0.28 \mathrm{~mm} / \mathrm{mo}$ at the middle DTA $(P<.001)$, and $-0.02 \pm 0.13,0.07 \pm 0.07$, and $0.16 \pm 0.14 \mathrm{~mm} / \mathrm{mo}$ at the distal DTA, respectively $(P<.001$; Table 1). Partial thrombosis correlated with a significantly greater growth rate in all 3 segments and was fastest in the proximal DTA. The growth rate of a completely patent false lumen was slower than that of a partial thrombosis but faster than that of a complete thrombosis. During the follow-up period, a trend was seen for regression in the false lumen size in some patients in the thrombosed false lumen group. We extracted this subgroup from the thrombosed category and defined it as a "regressed" group if the thickness of the thrombosed false lumen had regressed to $<2 \mathrm{~mm}$ or was absent. No difference was found in the growth rates between the complete thrombosis and regressed groups (Table E2). In addition, the 
TABLE 1. Segmental aortic growth rate according to segmental false lumen status of descending thoracic aorta

\begin{tabular}{|c|c|c|c|c|}
\hline Aortic segment & Completely thrombosed & Completely patent & Partially thrombosed & $P$ value \\
\hline Proximal DTA & $31(46.3)$ & $14(20.9)$ & $22(32.8)$ & \\
\hline Diameter on first postoperative CT scan (mm) & $37.59 \pm 5.82$ & $41.36 \pm 7.72$ & $43.96 \pm 8.39$ & .008 \\
\hline Growth rate $(\mathrm{mm} / \mathrm{mo})$ & & & & .001 \\
\hline Mean \pm SD & $-0.10 \pm 0.31$ & $0.09 \pm 0.22$ & $0.35 \pm 0.60$ & \\
\hline Range & -1.58 to 0.31 & -0.27 to 0.74 & -0.10 to 2.86 & \\
\hline Middle DTA & $32(47.8)$ & $17(25.4)$ & $18(26.9)$ & \\
\hline Diameter on first postoperative CT scan (mm) & $33.34 \pm 4.61$ & $33.60 \pm 5.62$ & $35.19 \pm 4.80$ & .434 \\
\hline Growth rate $(\mathrm{mm} / \mathrm{mo})$ & & & & $<.001$ \\
\hline Mean \pm SD & $-0.04 \pm 0.18$ & $0.12 \pm 0.19$ & $0.28 \pm 0.28$ & \\
\hline Range & -0.48 to 0.53 & -0.03 to 0.78 & -0.12 to 1.06 & \\
\hline Distal DTA & $25(37.3)$ & $17(25.4)$ & $25(37.3)$ & \\
\hline Diameter on first postoperative CT scan (mm) & $29.04 \pm 3.57$ & $30.48 \pm 4.60$ & $31.68 \pm 3.35$ & .054 \\
\hline Growth rate $(\mathrm{mm} / \mathrm{mo})$ & & & & $<.001$ \\
\hline Mean \pm SD & $-0.02 \pm 0.13$ & $0.07 \pm 0.07$ & $0.16 \pm 0.14$ & \\
\hline Range & -0.27 to 0.26 & 0.00 to 0.28 & -0.07 to 0.46 & \\
\hline
\end{tabular}

Data presented as n $(\%)$ or mean \pm standard deviation and range. DTA, Descending thoracic aorta; $C T$, computed tomography; $S D$, standard deviation.

partially thrombosed proximal DTA correlated with the largest baseline diameter (Table 1).

We also recategorized our patients according to their entire false lumen status instead of their independent segmental status. If a false lumen showed complete patency throughout the entire DTA, it would be considered completely patent; if a false lumen showed complete thrombosis or regression throughout the entire DTA, it was considered a complete thrombosis or regression, respectively. All others were considered partial thrombosis. By this definition, the distribution of cases between each subgroup changed, and the effect of partial thrombosis on the maximal growth rate was attenuated (Table 2).

\section{Reoperation}

The overall rate of freedom from distal aortic reoperation at 1,5 , and 10 years for the 155 patients who survived to discharge was $98 \%, 92 \%$, and $86 \%$ compared with $99 \%, 95 \%$, and $87 \%$ for the 67 patients included in the present study, respectively. Reoperations were required in 6 of 67 patients $(8.9 \%)$ during the follow-up period (median interval, 4.74 years; range, 1.00-11.28). All 6 patients underwent proximal DTA replacement because aneurysms had formed, including 1 thoracoabdominal-aorta replacement. All 6 patients (4 were in the proximal DTA partial thrombosis subgroup) survived reoperation. The freedom from reoperation rate according to the false lumen status of the proximal DTA at 1, 5, and 10 years was
$100 \%, 100 \%$, and $100 \%$ for complete thrombosis, $100 \%, 100 \%$, and $88 \%$ for completely patent, and $95 \%$, $83 \%$, and $62 \%$ for partial thrombosis $(P=.013)$, respectively (Figure 1, $A$ ).

The freedom from reoperation rate according to the false lumen status of the middle DTA at 10 years was $100 \%$ for complete thrombosis, $64 \%$ for completely patent, and $85 \%$ for partial thrombosis $(P=.052)$ The rate for the distal DTA at 10 years was $100 \%$ for complete thrombosis, $64 \%$ for completely patent, and $90 \%$ for partial thrombosis $(P=.086)$.

\section{Overall Late Survival}

The overall survival rate at 1,5 , and 10 years for the 155 patients who survived to discharge was $92 \%, 84 \%$, and $58 \%$ compared with $100 \%, 98 \%$, and $89 \%$ for the 67 patients included in the present study, respectively. The overall survival rate according to the false lumen status of the proximal DTA at 1,5 , and 10 years was $100 \%, 100 \%$, and $89 \%$ for complete thrombosis, $100 \%, 100 \%$, and $100 \%$ for completely patent, and $100 \%, 94 \%$, and $80 \%$ for partial thrombosis $(P=.293)$ respectively (Figure 1, $B)$. The difference in the overall survival curves according to the false lumen status for the other 2 aortic segments were also nonsignificant. For the middle DTA at 10 years, the overall survival was $89 \%$ for complete thrombosis, $85 \%$ for completely patent, and $94 \%$ for partial thrombosis $(P=.843)$. For the distal DTA, it was $87 \%$ for complete

TABLE 2. Maximal segmental aortic growth rate according to false lumen status of entire descending thoracic aorta

\begin{tabular}{|c|c|c|c|c|c|}
\hline Aortic segment & $\begin{array}{c}\text { Regressed } \\
(\mathrm{n}=11 ; 16.4 \%)\end{array}$ & $\begin{array}{c}\text { Completely thrombosed } \\
(\mathrm{n}=10 ; 14.9 \%)\end{array}$ & $\begin{array}{c}\text { Completely patent } \\
(\mathrm{n}=11 ; 16.4 \%)\end{array}$ & $\begin{array}{l}\text { Partially thrombosed } \\
\quad(\mathrm{n}=35 ; 52.2 \%)\end{array}$ & $P$ value \\
\hline Diameter on first postoperative CT scan (mm) & $36.39 \pm 5.62$ & $38.68 \pm 6.78$ & $40.00 \pm 7.41$ & $42.41 \pm 8.00$ & .106 \\
\hline Growth rate $(\mathrm{mm} / \mathrm{mo})$ & $-0.04 \pm 0.10$ & $-0.18 \pm 0.53$ & $0.12 \pm 0.22$ & $0.19 \pm 0.52$ & .101 \\
\hline
\end{tabular}

Data presented as mean \pm standard deviation. $C T$, Computed tomography. 
TABLE 3. Incidence and timing of dynamic change in false lumen status in the proximal descending thoracic aorta

\begin{tabular}{|c|c|c|c|c|}
\hline \multirow[b]{2}{*}{$\begin{array}{l}\text { First postoperative } \\
\text { false lumen status }\end{array}$} & \multicolumn{4}{|c|}{ Dynamic change in false lumen status } \\
\hline & $\begin{array}{c}\text { Completely } \\
\text { patent }\end{array}$ & $\begin{array}{c}\text { Partially } \\
\text { thrombosed }\end{array}$ & $\begin{array}{l}\text { Completely } \\
\text { thrombosed }\end{array}$ & Regressed \\
\hline \multicolumn{5}{|l|}{$\begin{array}{l}\text { Completely patent } \\
\qquad(\mathrm{n}=19)\end{array}$} \\
\hline $\mathrm{n}(\%)$ & $10(52.6)^{*}$ & $8(42.1) \dagger$ & $2(10.5) \ddagger$ & $0(0.0) \S$ \\
\hline Median & 42 & 9.0 & 29.7 & \\
\hline Range & $11.0-81.3$ & $6.6-13.3$ & $14.6-44.8$ & \\
\hline \multicolumn{5}{|l|}{$\begin{array}{l}\text { Partially thrombosed } \\
\qquad(\mathrm{n}=20)\end{array}$} \\
\hline n $(\%)$ & $1(5.0)$ & $11(55.0)^{*}$ & $7(35.0) \ddagger$ & $3(15.0) \S$ \\
\hline Median & 84.0 & 15.2 & 23.9 & 23.9 \\
\hline Range & & $11.9-61.1$ & $8.3-42.5$ & $11.2-75.8$ \\
\hline \multicolumn{5}{|l|}{$\begin{array}{l}\text { Completely thrombosed } \\
\qquad(\mathrm{n}=15)\end{array}$} \\
\hline $\mathrm{n}(\%)$ & $0(0.0)$ & $0(0.0)$ & $2(13.3)^{*}$ & $13(86.7) \S$ \\
\hline Median & & & 55.0 & 13.3 \\
\hline Range & & & $47.7-62.5$ & $9.9-83.8$ \\
\hline
\end{tabular}

Data presented as $\mathrm{n}(\%)$, unless otherwise noted; median and range data refer to follow-up times in months. *Unchanged false lumen status. $\dagger$ False lumen became partially thrombosed. ‡False lumen became completely thrombosed (median interval, 29.7 and 23.9 months). §False lumen regressed.

thrombosis, $85 \%$ for completely patent, and $96 \%$ for partial thrombosis $(P=.958)$.

\section{Dynamic Change in False Lumen Status}

Of the 54 patients available for an assessment of the dynamic changes in false lumen evolution, $52.6 \%$ of the completely patent group, $55 \%$ of the partially thrombosed group, and $13.3 \%$ of the completely thrombosed group had an unchanged false lumen status (Table 3). Of the completely patent group, $42.1 \%$ became partially thrombosed (Table 3). Of the completely patent group, $10.5 \%$ and of the partially thrombosed group, 35\% developed completely thrombosed false lumens at a median interval of 29.7 and 23.9 months, respectively (Table 3). Regression occurred in $15 \%$ of the partially thrombosed group and $86.7 \%$ of the completely thrombosed group within a median interval of 23.9 and 13.3 months, respectively (Table 3).

\section{DISCUSSION}

We used a segmental analysis method to assess the false lumen status independently at 3 levels of the DTA, in contrast to the definition of the entire aortic false lumen condition adopted in previous studies of partial thrombosis. ${ }^{9-11,13,14}$ We found that after a TAAAD had been repaired, residual segmental partial thrombosis led to a greater regional aortic growth rate than did a completely patent or complete or regressed thrombosis at all 3 DTA levels. It also predicted a greater reoperation rate at the proximal DTA. The overall 10-year survival rate, however, was not significantly different among the study groups.

The predisposing effects of a patent false lumen on aortic growth and adverse outcomes after surgically repaired TAAAD or TBAAD have been previously reported. ${ }^{1-9}$ However, these studies usually had an ill-defined partially thrombosed false lumen, or they had grouped patent and thrombosed false lumens. Fattori and colleagues ${ }^{9}$ reported that a patent false lumen after a surgically repaired TAAAD had a greater expansion rate than did a partially thrombosed false lumen. Furthermore, a partial thrombosis on the last follow-up CT scan after TBAAD was associated with a lower growth rate than was a patent false lumen in 1 study, ${ }^{13}$ which showed no difference in the reoperation rate. Although the study also reported that the growth rate in the sac-formation subtype was greater in the partial thrombosis group than in the completely patent group, the claim lacked statistical power because of the low prevalence of that subtype in the study population.

In contrast, Tsai and colleagues ${ }^{10}$ first reported partial thrombosis of the false lumen as a significant independent predictor of postdischarge mortality after TBAAD, with a relative mortality risk of 2.7 compared with a completely patent false lumen. Moreover, partial thrombosis of the false lumen was an independent predictor of a greater aortic growth rate after TBAAD. ${ }^{11}$ Song and colleagues ${ }^{14}$ also found that a partially thrombosed distal false lumen after surgically repaired TAAAD, not only caused a greater growth rate in the DTA, but also predicted a greater risk of reoperations and poor survival.

The effect of partial thrombosis was attenuated, however, when we recategorized our patients using the definition of the entire false lumen status, such as was used in other studies. This suggested that an overall judgment of the false lumen status might mask the effect of some subtypes of partial thrombosis in certain aortic segments. It also did not discriminate among the various lengths and distributions of false lumen thrombosis. The conflicting results of previous studies could be attributable to the heterogeneous patient groups, diverse presentations of partial thrombosis, and the analytical methods used.

We have shown that a segmental partial thrombosis of the false lumen, regardless of its proximal or distal false lumen status, led to a greater regional growth rate. Tsai and colleagues ${ }^{10}$ proposed that the greater intraluminal pressure in a partially thrombosed false lumen could explain its effect on the aortic growth rate. This, however, was contradicted by the finding that a partially thrombosed false lumen will not necessarily present with only 1 entry site. An additional entry site will decompress the pressure of the false lumen. In a false lumen with a single inlet, spinal or visceral branches originating from the false lumen could also act as decompression outlets. ${ }^{13}$ Studies of abdominal aortic aneurysms have suggested a relationship between 


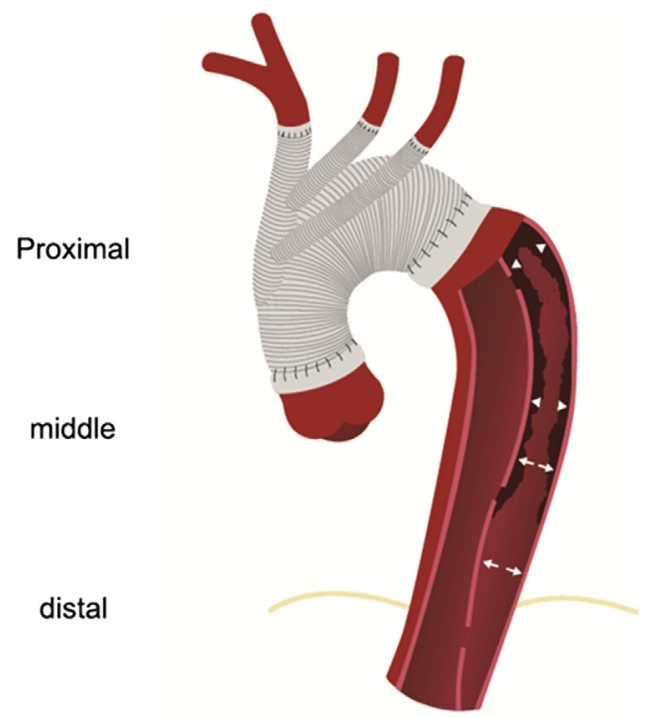

$\rightarrow$ effect of increased intra-luminal pressure

effect of partial thrombosis

FIGURE 2. A typical postoperative false lumen partial thrombosis pattern, with a single distal entry site and a proximal blind sac. In addition to the elevated intraluminal pressure (arrows), a supposed local effect of the mural thrombus (arrowheads) might explain the greater growth rate in the partially thrombosed portion than in the completely patent segment.

mural thrombosis and the risk of rupture because of hypoxia of the arterial wall, local inflammation, and localized wall weakening. ${ }^{12}$ A local effect of partial thrombosis, independent of the intraluminal pressure, could also exacerbate regional aortic expansion. The typical postoperative partially thrombosed false lumen pattern with a single distal entry site and a proximal blind sac will elevate the intraluminal pressure (Figure 2). The distribution of mural thrombi has been assumed to have a local effect on regional aortic growth (Figure 2). We hypothesized that the aortic segment with partial thrombosis would expand faster because it will be influenced by both the intraluminal pressure and the local mural thrombus effects. The lack of a local mural thrombus factor in the patent segment would explain why it grows more slowly than the partially thrombosed portion.

The baseline aortic size has been proved to be a predictor of aortic expansion. ${ }^{1,4,5,8}$ The DTA, especially in its proximal portion, has also been considered the fastest growing portion. ${ }^{2,6,9,13,14}$ Although the growth rate of the partially thrombosed group was fastest in every segment of the DTA, the additional effect of the baseline aortic size and proximal DTA on aortic growth made it possible to predict the need for a reoperation. These findings support the evolving trend of more extensive aortic reconstruction during the first operation for TAAAD, such as the frozen elephant trunk technique, to counteract the greatest risk of reoperation of the DTA. Uchida and colleagues ${ }^{15}$ reported that at 5 years postoperatively, the false lumen at the proximal descending aorta was patent in $29 \%$ of the ascending aortic or hemiarch replacement group but had thrombosed in all patients in the total arch replacement with a frozen elephant trunk group.

Our findings have also suggested that a partially thrombosed false lumen requires more frequent imaging surveillance. Earlier reintervention could be considered in this subgroup. However, the false lumen status can evolve during the follow-up period. ${ }^{2}$ More than $40 \%$ of the completely patent and partially thrombosed false lumens had a different false lumen status between their first postoperative and last follow-up CT scan. A trend was seen toward thrombus accumulation in these patients; however, the time required varied for each patient. Deciding on an earlier reintervention because of the findings from a first postoperative $\mathrm{CT}$ would be impractical. It would be more reasonable to consider earlier reintervention in patients whose partially thrombosed false lumen has persisted after a period of optimized antihypertensive control. From the observations in our small sample, if a partially thrombosed or complete patent false lumen had a chance of becoming completely thrombosed, it usually occurred within a median interval of 23.9 to 29.7 months. A more aggressive reintervention strategy could be considered beyond that period.

Our retrospective study was limited by the wide period of data collection, because it included patients treated with different surgical protocols. Our low follow-up rate $(43.2 \%)$ made our data less representative of the entire cohort. However, the freedom from reoperation rate at 10 years was not significantly different between the 155 patients who survived the initial operation and the 67 patients included in the present study. Our own definitions and analytical methods have made our results not directly comparable with similar studies. The size and location of the entry tears also influenced the fate of the false lumens. ${ }^{16}$ However, they were not completely identified in the present study, because we did not perform routine transesophageal echocardiography during the follow-up period. Additional analyses of the risk factors for growth rate and late outcomes are necessary to exclude other confounding factors.

\section{CONCLUSIONS}

Compared with other false lumen conditions, a segmental partially thrombosed false lumen after surgically repaired TAAAD predicted a greater regional aortic growth rate and late reoperation rate. The effect of diverse partial thrombosis patterns might be better explained using segmental analysis.

The authors thank Ms. Chao-Jung Shih for her excellent statistical analyses. 


\section{References}

1. Zierer A, Voeller RK, Hill KE, Kouchoukos NT, Damiano RJ Jr, Moon MR. Aortic enlargement and late reoperation after repair of acute type A aortic dissection. Ann Thorac Surg. 2007;84:479-86; discussion 486-7.

2. Park KH, Lim C, Choi JH, Chung E, Choi SI, Chun EJ, et al. Midterm change of descending aortic false lumen after repair of acute type I dissection. Ann Thorac Surg. 2009;87:103-8.

3. Fattouch K, Sampognaro R, Navarra E, Caruso M, Pisano C, Coppola G, et al. Long-term results after repair of type a acute aortic dissection according to false lumen patency. Ann Thorac Surg. 2009;88:1244-50.

4. Yeh $\mathrm{CH}$, Chen MC, Wu YC, Wang YC, Chu JJ, Lin PJ. Risk factors for descending aortic aneurysm formation in medium-term follow-up of patients with type A aortic dissection. Chest. 2003;124:989-95.

5. Sakaguchi G, Komiya T, Tamura N, Kimura C, Kobayashi T, Nakamura H, et al. Patency of distal false lumen in acute dissection: extent of resection and prognosis. Interact Cardiovasc Thorac Surg. 2007;6:204-7.

6. Halstead JC, Meier M, Etz C, Kimura C, Kobayashi T, Nakamura H, et al. The fate of the distal aorta after repair of acute type A aortic dissection. J Thorac Cardiovasc Surg. 2007;133:127-35.

7. Kimura N, Tanaka M, Kawahito K, Yamaguchi A, Ino T, Adachi H. Influence of patent false lumen on long-term outcome after surgery for acute type A aortic dissection. J Thorac Cardiovasc Surg. 2008;136:1160-6, 1166.e1-3.

8. Immer FF, Hagen U, Berdat PA, Eckstein FS, Carrel TP. Risk factors for secondary dilatation of the aorta after acute type A aortic dissection. Eur J Cardiothorac Surg. 2005;27:654-7.
9. Fattori R, Bacchi-Reggiani L, Bertaccini P, Napoli G, Fusco F, Longo M, et al. Evolution of aortic dissection after surgical repair. Am J Cardiol. 2000;86: 868-72.

10. Tsai TT, Evangelista A, Nienaber CA, Myrmel T, Meinhardt G, Cooper JV, et al. Partial thrombosis of the false lumen in patients with acute type B aortic dissection. N Engl J Med. 2007;357:349-59.

11. Trimarchi S, Tolenaar JL, Jonker FH, Murray B, Tsai TT, Eagle KA, et al. Importance of false lumen thrombosis in type B aortic dissection prognosis. J Thorac Cardiovasc Surg. 2013;145(3 Suppl):S208-12.

12. Song SW, Chang BC, Cho BK, Yi G, Youn YN, Lee S, et al. Effects of partial thrombosis on distal aorta after repair of acute DeBakey type I aortic dissection. J Thorac Cardiovasc Surg. 2010;139:841-7.e1; discussion 847.

13. Sueyoshi E, Sakamoto I, Uetani M. Growth rate of affected aorta in patients with type B partially closed aortic dissection. Ann Thorac Surg. 2009;88: 1251-7.

14. Vorp DA, Lee PC, Wang DH, Makaroun MS, Nemoto EM, Ogawa S, et al. Association of intraluminal thrombus in abdominal aortic aneurysm with local hypoxia and wall weakening. J Vasc Surg. 2001;34:291-9.

15. Uchida N, Shibamura H, Katayama A, Shimada N, Sutoh M, Ishihara H. Operative strategy for acute type a aortic dissection: ascending aortic or hemiarch versus total arch replacement with frozen elephant trunk. Ann Thorac Surg. 2009; 87:773-7.

16. Evangelista A, Salas A, Ribera A, Ferreira-Gonzalez I, Cuellar H, Pineda V, et al. Long-term outcome of aortic dissection with patent false lumen: predictive role of entry tear size and location. Circulation. 2012;125:3133-41. 


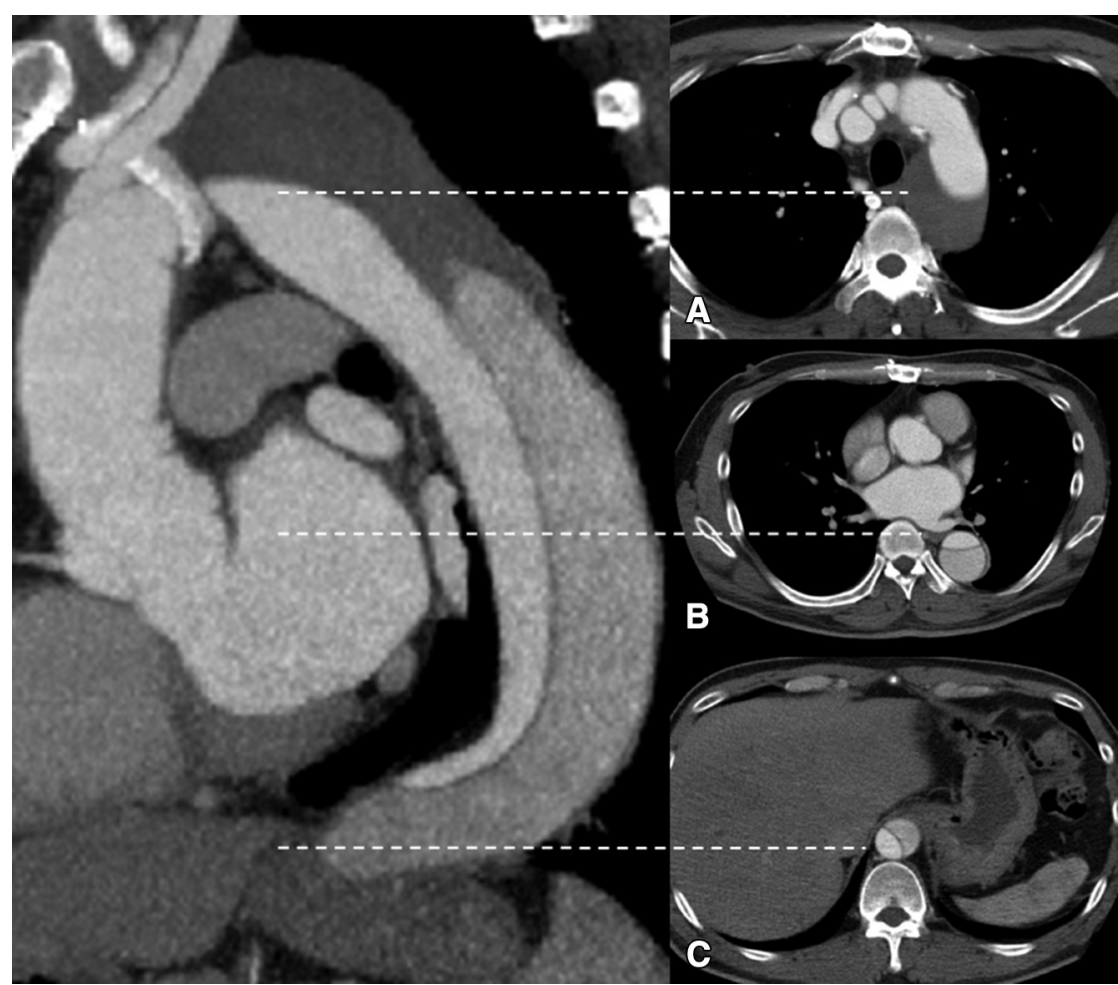

FIGURE E1. Sagittal view of a postoperative residual dissected false lumen considered partially thrombosed by judging the entire aorta. In contrast, we used a segmental assessment. A, The proximal descending thoracic aorta (DTA) distal to the left subclavian artery and aortic arch was classified as completely thrombosed. B, The middle DTA segment was classified as completely patent. C, The distal DTA at the level of the diaphragm crura was classified as completely patent. 
TABLE E1. Patient demographics and perioperative data

\begin{tabular}{|c|c|}
\hline Patient demographics & Value \\
\hline Age $(y)$ & $52.96 \pm 12.08$ \\
\hline Male gender & $50(74.6)$ \\
\hline Diabetes & $1(1.5)$ \\
\hline Hypertension & $49(73.1)$ \\
\hline Marfan syndrome & $4(6.0)$ \\
\hline End-stage renal disease* & $2(3.0)$ \\
\hline Coronary artery disease & $4(6.0)$ \\
\hline Old stroke & $1(1.5)$ \\
\hline Previous aortic dissection & $4(6.0)$ \\
\hline Previous aortic aneurysm & $6(9.0)$ \\
\hline \multicolumn{2}{|l|}{ Preoperative conditions } \\
\hline Malperfusion & $26(38.8)$ \\
\hline Syncope & $9(13.4)$ \\
\hline Stroke & $1(1.5)$ \\
\hline Shock & $11(16.4)$ \\
\hline Cardiopulmonary cerebral resuscitation & $4(6.0)$ \\
\hline Hemopericardium & $27(40.3)$ \\
\hline Tamponade & $8(11.9)$ \\
\hline Heart failure & $1(1.5)$ \\
\hline Respiratory insufficiency & $7(10.4)$ \\
\hline Hepatic failure & $7(10.4)$ \\
\hline Renal failure & $14(20.9)$ \\
\hline Aortic regurgitation & $18(26.9)$ \\
\hline \multicolumn{2}{|l|}{ Cannulation site } \\
\hline Axillary artery, antegrade cerebral perfusion & $48(71.6)$ \\
\hline Femoral artery, retrograde cerebral perfusion & $19(28.4)$ \\
\hline \multicolumn{2}{|l|}{ Proximal procedure } \\
\hline Ascending aorta only & $49(73.1)$ \\
\hline Ascending aorta + aortic valve resuspension & $5(7.5)$ \\
\hline Ascending aorta + aortic valve replacement & $1(1.5)$ \\
\hline Bentall procedure & $11(16.4)$ \\
\hline David procedure & $1(1.5)$ \\
\hline \multicolumn{2}{|l|}{ Distal procedure } \\
\hline No arch reconstruction & $27(40.3)$ \\
\hline Hemiarch reconstruction & $15(22.4)$ \\
\hline Subtotal arch reconstruction & $19(28.4)$ \\
\hline Total arch reconstruction & $6(9.0)$ \\
\hline Elephant trunk & $4(6.0)$ \\
\hline \multicolumn{2}{|l|}{ Location of inlet } \\
\hline Aortic root & $1(1.5)$ \\
\hline Ascending aorta & $31(46.3)$ \\
\hline Arch & $16(23.9)$ \\
\hline Descending aorta & $4(6.0)$ \\
\hline Ascending aorta plus arch & $4(6.0)$ \\
\hline Arch plus descending aorta & $1(1.5)$ \\
\hline
\end{tabular}


TABLE E2. Segmental aortic growth rate according to segmental false lumen status of descending thoracic aorta

\begin{tabular}{|c|c|c|c|c|c|}
\hline Aortic segment & Regressed & Completely thrombosed & Completely patent & Partially thrombosed & $P$ value \\
\hline Proximal DTA & $20(29.9)$ & $11(16.4)$ & $14(20.9)$ & $22(32.8)$ & \\
\hline Diameter on first postoperative CT scan (mm) & $36.77 \pm 6.51$ & $39.07 \pm 4.15$ & $41.36 \pm 7.72$ & $43.96 \pm 8.39$ & .015 \\
\hline Growth rate $(\mathrm{mm} / \mathrm{mo})$ & & & & & .003 \\
\hline Mean \pm SD & $-0.15 \pm 0.36$ & $-0.01 \pm 0.17$ & $0.09 \pm 0.22$ & $0.35 \pm 0.60$ & \\
\hline Range & -1.58 to 0.09 & -0.31 to 0.31 & -0.27 to 0.74 & -0.10 to 2.86 & \\
\hline Middle DTA & $16(23.9)$ & $16(23.9)$ & $17(25.4)$ & $18(26.9)$ & \\
\hline Diameter on first postoperative CT scan (mm) & $31.38 \pm 4.29$ & $35.31 \pm 4.15$ & $33.60 \pm 5.62$ & $35.19 \pm 4.80$ & .075 \\
\hline Growth rate $(\mathrm{mm} / \mathrm{mo})$ & & & & & $<.001$ \\
\hline Mean \pm SD & $-0.06 \pm 0.09$ & $-0.02 \pm 0.24$ & $0.12 \pm 0.19$ & $0.28 \pm 0.28$ & \\
\hline Range & -0.26 to 0.05 & -0.48 to 0.53 & -0.03 to 0.78 & -0.12 to 1.06 & \\
\hline Distal DTA & $15(22.4)$ & $10(14.9)$ & $17(25.4)$ & $25(37.3)$ & \\
\hline Diameter on first postoperative CT scan (mm) & $28.13 \pm 3.56$ & $30.41 \pm 3.28$ & $30.48 \pm 4.60$ & $31.68 \pm 3.35$ & .046 \\
\hline Growth rate $(\mathrm{mm} / \mathrm{mo})$ & & & & & $<.001$ \\
\hline Mean \pm SD & $-0.06 \pm 0.10$ & $0.04 \pm 0.16$ & $0.07 \pm 0.07$ & $0.16 \pm 0.14$ & \\
\hline Range & -0.24 to 0.06 & -0.27 to 0.26 & 0.00 to 0.28 & -0.07 to 0.46 & \\
\hline
\end{tabular}

Data presented as $\mathrm{n}(\%)$ or mean \pm standard deviation and range. $D T A$, Descending thoracic aorta; $C T$, computed tomography; $S D$, standard deviation. 